\section{Recherche clinique et sciences humaines et sociales à I'Institut National du Cancer (INCa)}

\section{Norbert Amsellem}

I'INCa ont investi le champ de la recherche clinique en développant une thématique consacrée aux conditions de développement des essais cliniques en cancérologie. Ce programme visait à répondre à l'exigence, explicitement formulée dans la mesure 67 du premier Plan cancer (2003-2007), d'augmenter l'inclusion des patients dans les essais cliniques ${ }^{1}$. L'appel à projets lancé en 2006 dans ce cadre a donné lieu au financement de quatre programmes de recherche. L'un de ces projets, coordonné par C. Julian-Reynier (UMR 912 Inserm, Institut Paoli-Calmettes) et actuellement en cours d'achèvement, portait précisément sur le déroulement et les enjeux pour les patients d'un essai de thérapie ciblée du cancer du sein fondé sur une signature génomique (essai SA02 et essai de validation du test MAMMAPRINT dans l'essai européen MINDACT). Les autres travaux financés proposaient une analyse sociologique comparée de la participation des médecins à la recherche clinique dans trois essais multicentriques randomisés (Patrick Castel, groupe de recherche en économie de la santé et réseaux de soins en cancérologie [GRESAC], CNRS/centre Léon Bérard, Lyon); une approche sociologique et sémiotique du consentement éclairé et de la compréhension de l'information des patients lors d'essais cliniques en oncologie » (Pascal Ducournau, centre interdisciplinaire de recherches urbaines et sociologiques - centre d'études des rationalités et des savoirs [CIRUS-CERS], CNRS - université de Toulouse-Le Mirail) ; enfin, une évaluation comparative d'une restitution collective versus individualisée des résultats des essais de recherche clinique aux patients (Dominique Genre, Institut Paoli-Calmettes, Marseille). Les années suivantes, plusieurs autres projets de recherche en SHS proposés par des chercheurs ont

\footnotetext{
${ }^{1}$ Extrait de la mesure 67 du Plan cancer : Donner une impulsion forte à la recherche sur le cancer, au travers de la définition d'une stratégie nationale de recherche et de financements d'appui à cette stratégie.

«Recherche clinique : évaluations de stratégies thérapeutiques, médicales, chirurgicales, radiothérapiques et études populationnelles :

- L'enjeu est ici de développer une recherche clinique coordonnée au niveau national par l'identification de programmes concertés entre les différents groupes coopérateurs. L'objectif, qui est d'atteindre à terme $10 \%$ de patients inclus dans les pôles régionaux de cancérologie et de viser $5 \%$ dans les autres structures doit concilier une qualité des études conforme aux standards internationaux et une accessibilité des protocoles à tout patient qui le désire. Dans cette action, les grands groupes coopérateurs seront, pour des raisons d'efficacité, considérés prioritairement par l'Institut du Cancer dans le financement des essais. En revanche, ces protocoles seront ouverts à l'inclusion le plus largement possible. »
}

Coordination des recherches

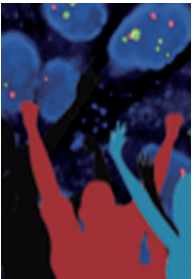
en sciences humaines et sociales, Institut National du Cancer (INCa), 52 , avenue André Morizet, 92513 Boulogne Billancourt Cedex, France. namsellem@institutcancer.fr

également contribué à l'analyse du développement des essais cliniques en cancérologie. Citons par exemple l'analyse médico-économique (coût-efficacité) d'un test fondé sur une signature génomique (Michaël Schwarzinger, Institut Gustave Roussy, Villejuif) ; l'étude de l'expérience et du statut des patients dans la recherche biomédicale en cancérologie en France (Bertrand Pulman, université Paris XIII), ou encore celle de l'impact psychologique et émotionnel de la participation aux études cliniques en oncologie médicale (Stéphanie Clisant, Véronique Christophe, centre Oscar Lambret, Lille).

Les nouvelles approches thérapeutiques consistent d'une part en des traitements ciblés sur des types ou sous-types de tumeurs plus précisément identifiées, et d'autre part concernent des groupes de patients (plutôt que des patients individuels stricto sensu) identifiés comme répondant à ces traitements et donc susceptibles de bénéficier des molécules innovantes. Cette évolution comporte de nouveaux enjeux pour les recherches en SHS sur le cancer. Par exemple, l'analyse des usages cliniques en routine de ces tests, différents des conditions de leurs essais de validation, et leurs conséquences pour les patients et pour la santé publique. Les traitements qui font actuellement l'objet d'essais cliniques en cancérologie comportent également un enjeu d'équité, de justice sociale et d'égalité d'accès aux innovations. Une médecine personnalisée - ce qui est encore un autre concept que celui de médecine individualisée - doit ainsi nécessairement prendre en compte les dimensions socio-économiques des conditions de vie et des ressources des patients afin de réduire les risques d'accroissement des inégalités sociales en matière de santé. C'est tout l'enjeu de la dimension éthique, économique, psychologique et sociale de la médecine personnalisée vers laquelle la cancérologie, entre autres, s'achemine à grands pas. $\diamond$

Clinical research and human and social sciences at Institut National du Cancer (INCa) 